\title{
Local and gauge invariant observables in gravity
}

\author{
Igor Khavkine \\ Department of Mathematics, University of Trento, and TIFPA-INFN, Trento, \\ I-38123 Povo (TN) Italy \\ E-mail: igor.khavkine@unitn.it
}

\begin{abstract}
It is well known that general relativity (GR) does not possess any non-trivial local (in a precise standard sense) and diffeomorphism invariant observables. We propose a generalized notion of local observables, which retain the most important properties that follow from the standard definition of locality, yet is flexible enough to admit a large class of diffeomorphism invariant observables in GR. The generalization comes at a small price, that the domain of definition of a generalized local observable may not cover the entire phase space of GR and two such observables may have distinct domains. However, the subset of metrics on which generalized local observables can be defined is in a sense generic (its open interior is non-empty in the Whitney strong topology). Moreover, generalized local gauge invariant observables are sufficient to separate diffeomorphism orbits on this admissible subset of the phase space. Connecting the construction with the notion of differential invariants, gives a general scheme for defining generalized local gauge invariant observables in arbitrary gauge theories, which happens to agree with well-known results for Maxwell and Yang-Mills theories.
\end{abstract}

PACS numbers: 04.20.-q, 04.20.Cv, 03.50.-z, 04.62.+v 


\section{Introduction}

The goal of this note is to outline a connection between the theory of differential invariants and local observables in gauge theories, in the sense of classical and quantum field theory. The main example we will treat is gravity, or more precisely general relativity (GR) possibly coupled to matter fields, which is a gauge theory with diffeomorphisms as the group of gauge transformations. The differential invariants in this case are essentially scalars that can be tensorially constructed from the Riemann curvature tensor and its covariant derivatives. The core idea of the connection to local observables appeared already in the proposal of Bergmann and Komar [2, 3]. However, it seems, that the idea has never been taken to the logical conclusion that we intend to sketch below.

Consider the theory of a, say scalar, field $\phi$ on an $n$-dimensional spacetime manifold $M$. The prototypical example of a local observable in this theory is a smeared field

$$
\phi(f)=\int_{M} \phi(x) f(x)
$$

where the smearing test function $f \in \Omega^{n}(M)$ is $C^{\infty}$ with compact support. Those last two properties are key to making $\phi(f)$ a useful observable. Classically, an observable $F: \Phi \mapsto F(\Phi)$ is a map from field configurations to real numbers. A smeared field acts as $\phi(f): \Phi \mapsto \int_{M} \Phi(x) f(x)$. The compactness of the support of $f$ makes sure that this integral converges for an arbitrary field configuration, so that $\phi(f)$ has a large domain of definition on the phase space of the theory (on all of it, in this case). The smoothness of $f$ makes sure that the Poisson bracket

$$
\{\phi(f), \phi(g)\}=\int_{M \times M} f(x) E(x, y) g(y),
$$

where $E(x, y)$ is the distributional kernel of the Peierls formula and $\phi(g)$ is a similar smeared field, is well defined as a distributional integral. Compact support also helps with the convergence of the Poisson bracket integral. Quantum mechanically, the field $\phi(x)$ is promoted to an operator valued distribution. The smoothness of the smearing function $f$ is then essential to get an honest (though unbounded) operator corresponding to $\phi(f)$. The expectation values of products of smeared fields like

$$
\langle\phi(f) \phi(g)\rangle=\int_{M \times M}\langle\phi(x) \phi(y)\rangle f(x) g(y),
$$

are also distributional integrals with respect to the 2-point singular kernel $\langle\phi(x) \phi(y)\rangle$. Thus, the smoothness of $f$ and $g$ are again necessary to make sure that this integral is locally well-defined (UV finite), with their compact support ensuring its global convergence (IR finiteness). In short, we say that the smoothness of test functions, like $f$, diffuses the $U V$ singularities of local fields, like $\phi(x)$, and their compact support IR regularizes them.

An immediate generalization is the notion of a multilocal observable, which is given by a formula of the form

$$
\int_{M^{m}} \phi\left(x_{1}\right) \cdots \phi\left(x_{m}\right) f\left(x_{1}, \ldots, x_{m}\right),
$$

where the smearing test function $f \in \Omega^{m n}\left(M^{m}\right)$ is $C^{\infty}$ with compact support. It should be noted that the Poisson bracket of two local observables, as defined by 
Equation (2), is in general no longer a local observable. Rather, as in the example of $\phi^{2}(f)=\int_{M} \phi^{2}(x) f(x)$, it is (almost) bilocal (multilocal with $l=2$ ),

$$
\left\{\phi^{2}(f), \phi^{2}(g)\right\}=\int_{M \times M} 2 \phi(x) f(x) E(x, y) 2 \phi(y) g(y),
$$

with the caveat that the smearing function $f(x) E(x, y) g(y)$ is a distribution and could be non-smooth. Thus, another natural generalization that invites itself is that of multilocal observables with distributional smearing, though the identification of the class of distributions that can be consistently allowed becomes rather technical. We mention these generalizations only for completeness, with the remainder of this note concentrating on local observables with smooth smearings. Though, we do briefly come back to multilocal observables in Sections 5 and 6 .

In the case when $\phi(x)$ is a local field in a gauge theory, another important property demanded of a local observable like $\phi(f)$ is gauge invariance. That is, the value of $\phi(f)$ (numerical value classically, and operatorial value quantum mechanically) stays invariant under the action of gauge transformations. Any physically meaningful quantity may only be represented by a gauge invariant observable. It is common knowledge that, in gravitational theories, the set of local gauge invariant observables is trivial (see for instance [16] or [7], for a clear discussion). Such a statement can of course be made once a suitably precise notion of locality and gauge invariance are given, as we do in Section 2. On the other hand, a slight relaxation of that standard notion of locality, which we propose in Section 3, opens the door to the introduction in Section 4 of a large class of gravitational observables that are gauge invariant (thanks to the use of differential invariants), diffuse UV singularities and are IR regularizing. Finally, we address the computation of Poisson brackets between generalized local gauge invariant gravitational observables in Section 5. Ultimately, we propose to treat this generalized notion as the true definition of local observables.

In the rest of the note we discuss only classical observables. Comments on how the constructions outlined below impact perturbative quantum field theory are left for the Discussion in Section 6, where we also mention various limitations and open problems of our proposal.

We finish this section with a brief historical remark. The idea of constructing observables in gravitational theories based on differential invariants (curvature scalars) first appeared clearly in the works of Bergmann and Komar [2, 3]. Unfortunately, they never published a computation of Poisson brackets for such observables. Such computations appeared first in the work of DeWitt [12], who used the Peierls bracket formalism. Since then, related ideas have appeared sporadically in the literature, more recently referred to as relational observables [34]. Some ideas in spirit similar to those presented below can also be found in [16] and [7], with the latter following-up a slightly different line of ideas that attempted to expand the notion of local obsrvables by modifying the notion of gauge invariance [31, 15].

\section{Standard local observables in field theories}

Let us briefly set up the geometric formalism of classical field theory. We will mostly follow the references $[21,23]$, with $[6,15,18,8]$ being complementary sources. We take $M$ to be an oriented $n$-dimensional smooth manifold. Usually one endows $M$ with a Lorentzian metric, but we are working at a level of generality where that is not 
necessary. Take a vector bundle $F \rightarrow M$, the field bundle, and denote its sections as $\Phi: M \rightarrow F$, a field configuration. In more generality, $F \rightarrow M$ could be a more general smooth bundle, but we will stick to the vector bundle case for simplicity.

By $\pi^{k}: J^{k} F \rightarrow M$, for $k=0,1, \ldots, \infty$, we denote the bundle of $k$-jets of the field bundle $F \rightarrow M$. Jetsł naturally and geometrically capture information about higher derivatives of sections of $F \rightarrow M$ over a point of $M$. Given a $k$-jet, throwing away all the information about order- $k$ derivatives gives a $(k-1)$-jet. In other words, we have natural bundle projections $\pi_{k-1}^{k}: J^{k} F \rightarrow J^{k-1} F$ over $M$, until we get $J^{0} F=F$. Any section $\Phi: M \rightarrow F$ can be naturally augmented with the information about its derivatives (its jet) at every point of $M$, thus defining the $k$-jet extension section $j^{k} \Phi: M \rightarrow J^{k} F$. To be more concrete, consider a fiber-adapted local coordinate system $\left(x^{i}, \phi^{a}\right)$ on $F$. It induces an adapted local coordinate system $\left(x^{i}, \phi_{I}^{a}\right)$ on $J^{k} F$ over that on $F$, where $I=\varnothing, i, i j, \ldots$ ranges all possible multi-indices. The coordinate system is adapted in the sense that the following identity holds for any field section $\Phi$ :

$$
\phi_{i_{1} \cdots i_{l}}^{a}\left(j^{k} \Phi(x)\right)=\partial_{i_{1}} \cdots \partial_{i_{l}} \phi^{a}(\Phi(x)) .
$$

Next, we introduce the field configuration space $\mathscr{C}=\Gamma(F)$, consisting of smooth sections of the vector bundle $F \rightarrow M$. It is an infinite dimensional vector space. It is convenient to endow it with the Whitney weak topology, which gives it the structure of a Fréchet space [20, 24]. Unfortunately the Whitney weak topology is too coarse for some of our purposes (its fundamental neighborhoods do not control the behavior of sections toward the open ends of non-compact manifolds), so we will mostly make use of the Whitney strong topology (see the discussion in Section 3). Further, the equations of motion of the field theory (e.g., Klein-Gordon equation for a scalar field, or Einstein's equations for the gravitational field) select the subspace of solutions, $\mathscr{P} \subset \mathscr{C}$, which we refer to as the (covariant) phase space. For non-linear equations, $\mathscr{P}$ is in general not a linear subspace of $\mathscr{C}$, however we will presume that $\mathscr{P}$ has a welldefined Fréchet manifold structure induced by its inclusion as a submanifold of the Fréchet space $\mathscr{C}$. We are ultimately interested in the algebra of observables $C^{\infty}(\mathscr{P})$. However, it is often more convenient to discuss elements of $C^{\infty}(\mathscr{P})$ as images of elements of $C^{\infty}(\mathscr{C})$ under the projection induced by the inclusion $\mathscr{P} \subset \mathscr{C}$. We make the simplifying assumption that this inclusion is sufficiently regular for the projection to be surjective. Then, strictly speaking, observables correspond to equivalence classes of elements of $C^{\infty}(\mathscr{C})$. However, we will not need to make use of this distinction below and may also refer to elements of $C^{\infty}(\mathscr{C})$ as observables, or alternatively as functionals.

On $\mathscr{C}$, we can define a special class of functions called local functionals (or observables) with the help of horizontal forms on $J^{k} F$. Horizontal forms, whose space we denote as $\Omega^{p, 0}(F, k) \subset \Omega^{p}\left(J^{k} F\right)$, are generated as linear combinations from the pullback $\left(\pi^{k}\right)^{*} \Omega^{p}(M)$ of forms on the spacetime with coefficients from $C^{\infty}\left(J^{k} F\right)$, meaning they are of the form $\alpha_{i_{1} \cdots i_{k}}\left(x^{i}, \phi_{I}^{a}\right) \mathrm{d} x^{i_{1}} \cdots \mathrm{d} x^{i_{k}}$. Of course, elements of $\Omega^{p, 0}(F, k)$ can be pulled back to $\Omega^{p, 0}(F, l)$ along the natural jet projections $J^{l} F \rightarrow J^{k} F$ for any $l>k$. It is convenient to take the increasing union (or direct limit) $\Omega^{p, 0}(F)=\bigoplus_{k=0}^{\infty} \Omega^{p, 0}(F, k) / \sim$, where the equivalence relation identifies a form in $\Omega^{p, 0}(F, k)$ with its pullback to any higher jet bundle, so that we do not need to worry about the order $k$ when it is not necessary. We call elements of $\Omega^{n, 0}(F)$ horizontal

$\ddagger$ Jets are a standard constructions in differential geometry. An introduction to jets, operations on them and their applications to differential equations can be found in [28]. See also the relevant appendices to $[21,23]$. 
densities. For any form $\alpha \in \Omega^{p}\left(J^{k} F\right)$, we define its spacetime support as the closure of the projection of its support onto $M, \operatorname{supp}_{M} \alpha=\overline{\pi^{k} \operatorname{supp} \alpha}$.

It is helpful to note that any form $\beta \in \Omega^{p}\left(J^{k} F\right)$ can be projected to a horizontal form $\mathrm{h}[\beta]=\alpha \in \Omega^{p, 0}(F, k+1)$, where the map acts as $\mathrm{h}\left[\mathrm{d} x^{i}\right]=\mathrm{d} x^{i}$ and $\mathrm{h}\left[\mathrm{d} \phi_{I}^{a}\right]=\phi_{I i}^{a} \mathrm{~d} x^{i}$ on coordinate forms, extends linearly and respects the wedge product. Another convenient operator to define is the Euler-Lagrange derivative $\delta_{E L}$ of a horizontal density $\alpha \in \Omega^{n, 0}(F)$. Locally, we define $\delta_{E L}[\alpha]$ by the following identity on $M$ :

$$
\left.\frac{\mathrm{d}}{\mathrm{d} t}\right|_{t=0}\left(j^{k}(\Phi+t \Psi)\right)^{*} \alpha(x)=\left(j^{k} \Phi\right)^{*} \delta_{E L}[\alpha]_{a}(x) \Psi^{a}(x)+\mathrm{d} \xi[\Phi ; \Psi],
$$

where each $\delta_{E L}[\alpha]_{a} \in \Omega^{n, 0}(F)$ and $\xi$ is some differential operator that depends linearly on its second argument. Globally, $\delta_{E L}[\alpha]$ is a horizontal density valued in the dual bundle $F^{*} \rightarrow M$. By the usual methods of variational calculus, this relation makes $\delta_{E L}[\alpha]$ unique and well-defined. All of these constructions, and more, naturally live in the context of the variational bicomplex [28], of which we shall not need to make further use in this note.

To any horizontal density $\alpha \in \Omega^{n, 0}(F)$ with compact spacetime support, we can associate a functional

$$
A[\Phi]=\int_{M}\left(j^{k} \Phi\right)^{*} \alpha .
$$

If, in local adapted coordinates, we have $\alpha=\tilde{\alpha}\left(x^{i}, \phi^{a}, \phi_{i}^{a}, \phi_{i j}^{a}, \ldots\right) \mathrm{d}^{n} x$, then

$$
A[\Phi]=\int_{M} \tilde{\alpha}\left(x^{i}, \phi^{a}(\Phi(x)), \partial_{i} \phi^{a}(\Phi(x)), \partial_{i} \partial_{j} \phi^{a}(\Phi(x)), \ldots\right) \mathrm{d}^{n} x .
$$

It is straightforward to verify that, by the compact spacetime support condition, the above integral converges for an arbitrary field configuration $\Phi \in \mathscr{C}$ and in fact $A \in C(\mathscr{C})$. Of course, we would like $A$ to be not only continuous, but also in some sense smooth on the infinite dimensional manifold $\mathscr{C}$. It is in fact possible to make use of an infinite dimensional calculus on Fréchet manifolds such that $A \in C^{\infty}(\mathscr{C})[24,15,8]$. We will not enter into such details, and simply declare functions like $A$ to be in $C^{\infty}(\mathscr{C})$. The class of functions on $\mathscr{C}$ defined by an equation like (8) will be referred to as local functionals.

On the other hand, given an element $A \in C^{\infty}(\mathscr{C})$, we can define a notion of spacetime support that can be attributed directly to $A$. If $A$ is local and comes from a horizontal density $\alpha$, there will of course be a relation between these two notions of support. More precisely, we define [6, Eq.5.22]

$$
\begin{aligned}
\operatorname{supp} A=\{x \in M \mid \forall \text { open } U \ni x \exists \Phi, \Psi & \in \mathscr{C}: \\
& \operatorname{supp} \Psi \subseteq U \text { and } A[\Phi+\Psi] \neq A[\Phi]\},
\end{aligned}
$$

which is always closed. In words, for any point $y \in M$ outside $\operatorname{supp} A$, there is a sufficiently small neighborhood $V \ni y$ so that any perturbation $\Psi$ of the argument of $A[\Phi]$ with supp $\Psi \subseteq V$ must leave the numerical value of $A$ unchanged, that is, $A[\Phi+\Psi]=A[\Phi]$. In other words, $A[\Phi]$ does not depend on the value of $\Phi$ in some neighborhood of $y$.

As mentioned above, we can give a precise relation between the spacetime support of a horizontal density and that of the corresponding local functional. Recall the 
Euler-Lagrange derivative $\delta_{E L}[\alpha]$ of a horizontal density $\alpha$ defined by Equation (7). Since $\delta_{E L}[\alpha]$ is not strictly speaking a horizontal density, we extend to it the notion of spacetime support so that $\operatorname{supp}_{M} \delta_{E L}[\alpha]$ is the union of the spacetime supports $\operatorname{supp}_{M} \delta_{E L}[\alpha]_{a}$ of its components.

Lemma 2.1. Let $\alpha \in \Omega^{n, 0}(F)$ be a horizontal density with compact spacetime support and $A[\Phi]=\int_{M}\left(j^{k} \Phi\right)^{*} \alpha$. Then

$$
\operatorname{supp}_{M} \delta_{E L}[\alpha] \subseteq \operatorname{supp} A \subseteq \operatorname{supp}_{M} \alpha .
$$

Proof. The second inclusion is trivial, because $\left(j^{k}(\Phi+\Psi)\right)^{*} \alpha=\left(j^{k} \Phi\right)^{*} \alpha$ whenever $\operatorname{supp} \Psi$ is outside of $\operatorname{supp}_{M} \alpha$, since the restriction of both sides of the equality to supp $\Psi$ is simply zero. The rest, namely supp $A \subseteq \operatorname{supp}_{M} \alpha$, follows from the defining Equation (10).

On the other hand, suppose that $p \in \operatorname{supp} \delta_{E L}[\alpha] \subseteq J^{k} F$. Then, we can always find a section $\Phi \in \mathscr{C}$ such that $j^{k} \Phi(x)=p$, where $x=\pi^{k}(p) \in \operatorname{supp}_{M} \delta_{E L}[\alpha]$. Since by construction $\left(j^{k} \Phi\right)^{*} \delta_{E L}[\alpha](x) \neq 0$, for each open $U \ni x$ there must exist a (without loss of generality compactly supported) $\Psi \in \mathscr{C}$ with supp $\Psi \subseteq U$ such that

$$
\int_{M}\left(j^{k} \Phi\right)^{*} \delta_{E L}[\alpha]_{a}(x) \Psi^{a}(x) \neq 0
$$

Therefore, by continuity in $t$, the formula in Equation (7) tells us that there must exist a $t \neq 0$, however small, such that $A[\Phi+t \Psi] \neq A[\Phi]$. That concludes the proof that $\operatorname{supp}_{M} \delta_{E L}[\alpha] \subseteq \operatorname{supp} A$.

\section{Generalized local observables}

A precise notion of a local functional on the space $\mathscr{C}$ of field configurations on a field bundle $F \rightarrow M$ was given in Section 2. This notion is plenty sufficient to identify a rich set of observables in the usual relativistic field theories, including gauge theories like Maxwell electrodynamics and Yang-Mills theory, but notably excluding gravitational theories like GR or GR with matter fields. The reason gravitational theories are different is because, as will be discussed in Section 4, the intersection between the space of local functionals and gauge invariant functionals on $\mathscr{C}$ is trivial (it consists only of constant functions). On the other hand, we can relax the above notion of locality in a precise way, without sacrificing much in the way of the physical motivation that lead to it, such that the new class of generalized local functionals does admit a rich set of gauge invariant observables even in gravitational theories. We discuss this precise generalized notion of locality below and leave the applications to gravitational theories to Section 4.

The two main properties of local functionals that we would like to relax are the (a) global domain of definition and (b) field independent compactness of support. We explain both of these properties and how they could be relaxed below.

Any element $A \in C^{\infty}(\mathscr{C})$, by definition, gives a well-defined value $A[\Phi]$ for any $\Phi \in \mathscr{C}$. That is, the domain of definition of $A$ is all of $\mathscr{C}$ (it is global). Imagine, on the other hand, that $A$ is defined only on a subset $\mathscr{U} \subseteq \mathscr{C}$. Could then $A$ still play the role of a physically meaningful observable? The answer is a qualified yes, provided $\mathscr{U}$ is sufficiently large, for example an open set. Such a restriction may be necessary if, for instance, we have precise control only over solutions that are not too 
distant from a reference solution, $\S$ some $\Phi \in \mathscr{U}$. At the classical level, having $A$ and $B$ defined on an open neighborhood $\mathscr{U} \ni \Phi$ is sufficient to compute their Poisson brackets $\|$ at $\Phi$ because that involves only local, differential operations. Perturbative QFT about $\Phi$ will also not be sensitive to anything outside an arbitrary neighborhood. Eventually, a non-perturbative formulation of a QFT would likely require observables to be globally defined. However, even then, we are likely to be interested in quantum states that (e.g., in a phase space formulation of quantum theory) would assign negligible weight to solutions outside a neighborhood $\mathscr{U}$ of some reference solution $\Phi$. To accommodate such an eventual situation, we could globalise the domain of definition of $A \in C^{\infty}(\mathscr{C})$ by extending it in an arbitrary, though controlled way, to all of $\mathscr{C}$ using standard geometric tools, like the Tietze extension and Steenrod-Wockel approximation theorems [37].

Given that we would like the domain $\mathscr{U} \subset \mathscr{C}$ of a generalized local functional to be open, it is important to reflect on the topology that we use on $\mathscr{C}$. Technical details on various topologies on function spaces can be found in the references [20, 24]. It was stated in the Introduction that it is conventional to endow $\mathscr{C}=\Gamma(F)$ with the Whitney weak topology, whose open sets are generated by those of the form

$$
\mathscr{U}_{K, U}^{k}=\left\{\Phi \in \Gamma(F) \mid j^{k} \Phi(K) \subseteq U\right\}
$$

where $k \geq 0, K \subseteq M$ is compact and $U \subseteq J^{k} F$ is open. The big disadvantage of the weak topology is that its neighborhoods cannot control the behavior of a section outside of a compact subset of the spacetime $M$, as we will need to do in the sequel. However, except in some cases when boundaries are present, the spacetimes that are of physical interest are non-compact. For example, any globally hyperbolic spacetime must be of the form $M \cong \mathbb{R} \times \Sigma$. An alternative topology is the Whitney strong topology, whose open sets are generated by those of the form

$$
\mathscr{U}_{U}^{k}=\left\{\Phi \in \Gamma(F) \mid j^{k} \Phi(M) \subseteq U\right\},
$$

where $k \geq 0$ and $U \subseteq J^{k} F$ is open. The big disadvantage of the strong topology is that it is incompatible with the structure of a topological vector space on $\mathscr{C}$ (multiplication by scalars fails to be continuous), let alone a Fréchet or any other kind of manifold structure. Note, though, that since our manifolds can be exhausted by compact sets, any open set in the strong topology is at worst a $G_{\delta}$ set in the weak topology (a countable intersection of open sets). Fortunately, there are many intermediate topologies between the weak and the strong that both allow a Fréchet structure and control the behavior of sections on all of $M$. One example is a variation on the strong topology that allows only those open $U \subseteq J^{k} F$ that have "uniform" vertical size over $M$ with respect to some connection, such as one induced by an auxiliary Riemannian metric. Another possibility is to add a compactifying boundary to $M$ and restrict our attention only those sections that extend in some nice way to the boundary, $\uparrow$ then

$\S$ An example of this kind is the celebrated result of Christodoulou and Klainerman [9] on the stability of Minkowski space in GR. Their result essentially constructs an open neighborhood $\mathscr{U}$ of the Minkowski metric on the phase space $\mathscr{P}$ of GR on $\mathbb{R}^{4}$ with asymptotically flat boundary conditions. On the other hand, we still have very little information about $\mathscr{P}$ outside that neighborhood.

\| Strictly speaking, Poisson brackets are expected to be defined only upon restriction to the phase space $\mathscr{P} \subset \mathscr{C}$. However, it is sometimes possible to lift Poisson brackets even to $\mathscr{C}$. This will be discussed in more detail in Section 5

I Perhaps the simplest implementation of this idea is to consider a piece of a globally hyperbolic spacetime that is bounded by two compact Cauchy surfaces as a compact spacetime in its own right with the future and past Cauchy surfaces as its boundaries. 
using the weak topology on that subspace with respect to the compactified spacetime $M$. However, it does not seem that there is an a priori canonical choice of such an intermediate topology and that the choice must be made in a way that is compatible with the behavior of solutions of the equations of motion of the theory. Note that a similar discussion, and in a related context, can be found in Section 5.2.1 of [21].

Being pragmatic, we stick to the Whitney strong topology for the remainder of this note, despite its drawbacks. The working hypothesis is that the results that will be found in the sequel, and the methods used to obtain them, will naturally generalize to the appropriate choice of intermediate topology.

Next, having taken the liberty of considering functionals that are defined only on open subsets $\mathscr{U} \subseteq \mathscr{C}$, let us consider the difference between the spacetime supports of a functional $A \in C^{\infty}(\mathscr{C})$ and its restriction $\left.A\right|_{\mathscr{U}} \in C^{\infty}(\mathscr{U})$. We can reasonably define supp $\left.A\right|_{\mathscr{U}}$ by replacing $\mathscr{C}$ with $\mathscr{U}$ in the definition (10). The logical quantifiers are arranged such that $\left.\operatorname{supp} A\right|_{\mathscr{U}} \subseteq \operatorname{supp} A$. In fact, we can define the even finer notion of spacetime support at $\Phi$ with respect to $\mathscr{U}$ given by

$$
\begin{aligned}
& \left.\operatorname{supp}_{\Phi} A\right|_{\mathscr{U}}=\{x \in M \mid \forall \text { open } U \ni x \exists(\Phi+\Psi) \in \mathscr{U}: \\
& \operatorname{supp} \Psi \subseteq U \text { and } A[\Phi+\Psi] \neq A[\Phi]\},
\end{aligned}
$$

which is also always closed. A further refinement is the notion of spacetime support at $\Phi$ given by

$$
\operatorname{supp}_{\Phi} A=\left.\bigcap_{\mathscr{U}} \operatorname{supp}_{\Phi} A\right|_{\mathscr{U}}
$$

with the intersection taken over all open neighborhoods $\mathscr{U} \ni \Phi$ such that $A$ is defined on $\mathscr{U}$. The distinction is that while $\left.\operatorname{supp}_{\Phi} A\right|_{\mathscr{U}}$ depends on the domain $\mathscr{U}$, $\operatorname{supp}_{\Phi} A$ only depends on the germ of $A$ at $\Phi$. Then $\left.\bigcup_{\Phi \in \mathscr{U}} \operatorname{supp}_{\Phi} A \subseteq \operatorname{supp} A\right|_{\mathscr{U}}$ and $\left.\operatorname{supp} A\right|_{\mathscr{U}}=\left.\bigcup_{\Phi \in \mathscr{U}} \operatorname{supp}_{\Phi} A\right|_{\mathscr{U}}$. So, clearly, supp $\left.A\right|_{\mathscr{U}}$ may fail to be compact, even if each individual $\left.\operatorname{supp}_{\Phi} A\right|_{\mathscr{U}}$ or $\operatorname{supp}_{\Phi} A$ is.

For $\left.A\right|_{\mathscr{U}}$ to be IR regularizing, as discussed in the Introduction, it suffices that the spacetime supports $\operatorname{supp}_{\Phi} A$ be compact for each $\Phi \in \mathscr{U}$. Thus, the much stronger condition of compact $\left.\operatorname{supp} A\right|_{\mathscr{U}}$ for an observable $\left.A\right|_{\mathscr{U}}$, while obviously sufficient for IR regularity, is not necessary. Such a relaxation of the requirements on the fielddependent spacetime support of observables was previously considered in [21, Sec.5.3.5] (see also [32]).

At the level of local functionals, we can relax the notion of locality given in Section 2 in the following way. Let $\Phi \in \mathscr{C}$ be a field configuration and $\alpha \in \Omega^{n, 0}(F)$ be a horizontal density such that the intersection $j^{k} \Phi(M) \cap \operatorname{supp} \alpha \subseteq J^{k} F$ is compact. Then we call the functional

$$
A[\Psi]=\int_{M}\left(j^{k} \Psi\right)^{*} \alpha
$$

a generalized local functional (or observable) at $\Phi$. The following result makes precise the way in which the properties of the functional $A$ fit with the preceding discussion.

Theorem 3.1. With $\Phi$ and $\alpha$ as above, there exists an open $\mathscr{U} \subseteq \mathscr{C}$ (in the strong topology) with $\Phi \in \mathscr{U}$ such that, for all $\Psi \in \mathscr{U}$, the integral in (17) is convergent and both $\left.\operatorname{supp}_{\Psi} A\right|_{\mathscr{U}}$ and $\operatorname{supp}_{\Psi} A$ are compact.

Proof. Pick a compact neighborhood $Q$ of $K=\pi^{k}\left(j^{k} \Phi(M) \cap \operatorname{supp} \alpha\right)$ and an open neighborhood $U \subset J^{k} F$ of $j^{k} \Phi(M \backslash Q)$ that does not intersect supp $\alpha$. Let $\mathscr{U} \subseteq \mathscr{C}$ be 
the set of all sections $\Psi: M \rightarrow F$ such that $j^{k} \Psi(M \backslash Q) \subset U$. Clearly, $\Phi \in \mathscr{U}$ and, by the definition of the Whitney strong topology, $\mathscr{U}$ is open. By construction, for any $\Psi \in \mathscr{U}$, we have $\left(j^{k} \Psi\right)^{*} \alpha=0$ on $M \backslash Q$. This means that $\operatorname{supp}\left[\left(j^{k} \Psi\right)^{*} \alpha\right] \subseteq Q$ and is itself compact (by virtue of being a closed subset of a compact set) and hence the integral defining $A[\Psi]$ is convergent. Finally, from the definition of $\mathscr{U}$, any point $x \in M \backslash Q$ has a neighborhood $V \subseteq M \backslash Q$ such that any $\Delta$ that has supp $\Delta \subseteq V$ and with $\Psi+\Delta \in \mathscr{U}$ and must satisfy $j^{k}(\Psi+\Delta)^{*} \alpha=0$ on $V$ and hence $A[\Psi+\Delta]=A[\Psi]$. Therefore $\left.\operatorname{supp}_{\Psi} A\right|_{\mathscr{U}} \subseteq Q$ and hence is itself compact. Its subset $\left.\operatorname{supp}_{\Psi} A \subseteq \operatorname{supp}_{\Psi} A\right|_{\mathscr{U}}$ is closed and hence also compact.

\section{Gauge invariance and local observables in gravitational theories}

GR is the theory of a Lorentzian metric field $G$, so that $F=S^{2} T^{*} M$, with the equation of motion (Einstein equation) specified by the Einstein-Hilbert Lagrangian, $\mathcal{L}[G]=R[G] \operatorname{vol}_{G}$, where $R[G]$ is the Ricci scalar and $\operatorname{vol}_{G}$ is the metric volume form. This Lagrangian also determines the gauge symmetries of the theory, which consist of diffeomorphisms of $M$ acting by pullback on metrics, $G \mapsto \chi^{*} G$ for a diffeomorphism $\chi: M \rightarrow M$. Thus, the physical (or reduced) phase space of GR is the quotient $\overline{\mathscr{P}}=\mathscr{P} / \mathscr{G}$, where $\mathscr{P} \subset \mathscr{C}$ is the set of solutions of Einstein equations (usually also taken to be globally hyperbolic) and $\mathscr{G}$ is the group of gauge transformations (diffeomorphisms of $M$ ). The observables that we are really interested in are those that constitute the algebra $C^{\infty}(\overline{\mathscr{P}})$. As before, it is convenient to use the quotient map $\mathscr{P} \rightarrow \overline{\mathscr{P}}$ to identify $C^{\infty}(\overline{\mathscr{P}}) \subset C^{\infty}(\mathscr{P})$ with those observables that are invariant under the action of the group $\mathscr{G}$ of gauge transformations. We refer to any element $A \in C^{\infty}(\mathscr{P})$, or $C^{\infty}(\mathscr{C})$, as a gauge invariant observable (or functional) if it is left invariant by the action of $\mathscr{G}$, that is, $A\left[\chi^{*} G\right]=A[G]$ for any diffeomorphism $\chi: M \rightarrow M$. For our purposes, a gravitational theory is a field theory that involves a metric tensor $G$ (though possibly other fields as well) and has the diffeomorphism group as the group $\mathscr{G}$ of gauge transformations. Clearly, GR is the representative example of a gravitational theory, but GR coupled to matter fields also falls into the same category. We will only consider pure GR below, but the discussion will also apply to more general gravitational theories.

It is a well-known folk result that GR does not have any local and gauge invariant observables in the standard sense of locality discussed in Section 2. However, the main observation of this note is that there in fact do exist local and gauge invariant observables in the generalized sense discussed in Section 3. The non-existence argument is pretty straight forward. Let $\alpha$ be a horizontal density on $k$-jets with $\operatorname{supp}_{M} \alpha$ compact and hence $A[G]=\int_{M}\left(j^{k} G\right)^{*} \alpha$ a local observable. A diffeomorphism $\chi: M \rightarrow M$ acts on it as

$$
(\chi A)[G]=A\left[\chi^{*} G\right]=\int_{M}\left(j^{k}\left(\chi^{*} G\right)\right)^{*} \alpha=\int_{M}\left(j^{k} G\right)^{*}\left[\left(p^{k} \chi^{*}\right)^{*} \alpha\right],
$$

where $p^{k} \chi^{*}: J^{k} F \rightarrow J^{k} F$ is the natural $k$-jet prolongation of the pullback action of a diffeomorphism on metrics $\chi^{*}: F \rightarrow F$. Clearly, the spacetime support of $\alpha$ transforms $\operatorname{as~supp}_{M}\left[\left(p^{k} \chi^{*}\right)^{*} \alpha\right]=\chi\left(\operatorname{supp}_{M} \alpha\right)$. Thus, by Lemma 2.1, the supp $\chi A$ moves around on $M$ under the action of diffeomorphisms. So, since we can choose $\chi$ such that $\operatorname{supp} A$ and supp $\chi A$ do not coincide, the functionals $A$ and $\chi A$ themselves cannot coincide. In particular, no observable $A$ can be gauge invariant if its spacetime support is 
different from $M$ (diffeomorphisms act on $M$ transitively). Spacetime manifolds of physical interest are never compact, hence no local observable (with, by definition from Section 2, compact spacetime support) can be gauge invariant. Colloquially, this is phrased as follows: gauge transformations of gravitational theories move spacetime points. This property is in contrast with gauge theories of Maxwell or Yang-Mills type, where gauge transformations leave intact the spacetime support of observables, thus allowing local observables to be gauge invariant.

We now give an explicit example of a functional that is both gauge invariant and local in the generalized sense. Subsequently, we will outline a general method for constructing more examples of a similar kind. Let us restrict for the moment the dimension $\operatorname{dim} M=4$. We will construct a horizontal density $\alpha \in \Omega^{4,0}(F)$ on $J^{3} F$. Let $W_{a b c d}=W_{a b c d}[G]$ and $\varepsilon_{a b c d}=\varepsilon_{a b c d}[G]$ denote respectively the Weyl and Levi-Civita tensors of the metric $G$. Then, define the dual Weyl tensor $W_{a b}^{*} c d=W_{a b c^{\prime} d^{\prime}} \varepsilon^{c^{\prime} d^{\prime} c d}$ and also the following curvature scalars

$$
\begin{array}{ll}
b^{1}=W_{a b}{ }^{c d} W_{c d}{ }^{a b}, & b^{3}=W_{a b}{ }^{c d} W_{c d}{ }^{e f} W_{e f}^{a b}, \\
b^{2}=W_{a b}{ }^{c d} W_{c d}^{* a b}, & b^{4}=W_{a b}{ }^{c d} W_{c d}{ }^{e f} W_{e f}^{* a b} .
\end{array}
$$

We have essentially defined maps $b=\left(b^{1}, b^{2}, b^{3}, b^{4}\right): J^{k} F \rightarrow \mathbb{R}^{4}$, for any $k \geq 2$. We will also use the notation $\left(b^{i}\right)$ for the standard global coordinates on this target $\mathbb{R}^{4}$. It is sufficient for us to take $k=3$ because we then want to define the horizontal density $\beta=\mathrm{h}\left[\mathrm{d} b^{1} \wedge \mathrm{d} b^{2} \wedge \mathrm{d} b^{3} \wedge \mathrm{d} b^{4}\right] \in \Omega^{4,0}(F, 3) \subset \Omega^{4,0}(F)$. Choose a point $r \in \mathbb{R}^{4}$, and a function $f \in C^{\infty}\left(\mathbb{R}^{4}\right)$ with compact support, such that $r \in \operatorname{supp} f$ but $\operatorname{supp} f \operatorname{does}$ not intersect any of the planes $b^{i}=0$. Finally, we define the desired horizontal density $\alpha=f(b) \beta \in \Omega^{4,0}(F)$, which gives rise to the functional

$$
A[G]=\int_{M}\left(j^{k} G\right)^{*} \alpha=\int_{M}\left(j^{k} G\right)^{*}\left(f\left(b^{1}, b^{2}, b^{3}, b^{4}\right) \mathrm{h}\left[\mathrm{d} b^{1} \wedge \mathrm{d} b^{2} \wedge \mathrm{d} b^{3} \wedge \mathrm{d} b^{4}\right]\right) .
$$

By construction, $\alpha$ satisfies two important properties. First, there is a non-empty open set $\mathscr{U} \subseteq \mathscr{C}$ (in the strong topology) such that the form $\left(j^{k} G\right)^{*} \alpha$ is smooth and has compact support on $M$ for any $G \in \mathscr{U}$. Thus, $A[G]$ is well-defined on $\mathscr{U}$ and hence constitutes a generalized local observable in the sense of Section 3 . The existence of such a domain $\mathscr{U}$ follows from a general result that will be discussed in Theorem 4.2 (see also the comments thereafter). Second, $A[G]$ is invariant under the action of diffeomorphisms. That is, $\left(p^{k} \chi^{*}\right)^{*} \alpha=\alpha$ for any diffeomorphism $\chi: M \rightarrow M$, which implies $A\left[\chi^{*} G\right]=A[G]$ for any $G$ on which the defining integral converges. The last invariance identity has to be used with a little bit of care, in that it only makes sense when both $G$ and $\chi^{*} G$ belong to $\mathscr{U}$, the domain of definition of $A$. Since, a priori $\mathscr{U}$ is not guaranteed to be itself diffeomorphism invariant, that condition may not be satisfied for an arbitrary $G \in \mathscr{U}$. One way to get around this issue is to, very reasonably, declare $A$ to be invariant under diffeomorphisms if $A\left[\chi^{*} G\right]=A[G]$ whenever both $G, \chi^{*} G \in \mathscr{U}$. Another way is to simply enlarge the domain to $\mathscr{U}^{\prime} \supseteq \mathscr{U}$ to the smallest diffeomorphism invariant domain that contains $\mathscr{U}$. Clearly, if $A$ is well defined on $\mathscr{U}$ it is also well defined on $\mathscr{U}^{\prime}$. A note of caution for second approach: while $\operatorname{supp}_{\Phi} A$, for any $\Phi \in \mathscr{U}$, is not altered by extending $A$ from $\mathscr{U}$ to $\mathscr{U}^{\prime}$, the inclusion $\left.\left.\operatorname{supp}_{\Phi} A\right|_{\mathscr{U}} \subseteq \operatorname{supp}_{\Phi} A\right|_{\mathscr{U}}$ ' may be strict, with $\left.\operatorname{supp}_{\Phi} A\right|_{\mathscr{U}}$, possibly failing to be compact even if $\left.\operatorname{supp}_{\Phi} A\right|_{\mathscr{U}}$ is.

In other words $\left.A\right|_{\mathscr{U}} \in C^{\infty}(\mathscr{U})$ is a local and gauge invariant observable in the generalized sense of Section 3 . 
The idea of using the curvature scalars $b^{i}$ to define observables in pure gravity goes back to the proposal of Komar and Bergmann [2, 3]. However, these authors, as well as many subsequent ones who came back to this idea (see [34] and references therein), intended to use $b^{i}$ as independent coordinates and simply express all other fields in terms of them. However, the resulting observables were often too singular in the sense discussed in the Introduction, since they would correspond to something like replacing our test function $f$ with a $\delta$-distribution. On the other hand, our addition of the integral and the smooth compactly supported function $f$ and the definition (20) provides the diffusion of UV singularities and the IR regularization, again discussed in the Introduction, that are needed in the contexts of QFT and classical Poisson structure.

The key ingredients in the above construction were the facts that we could choose the horizontal density $\alpha \in \Omega^{n, 0}(F)$ to be invariant under the prolonged action of diffeomorphisms on $J^{k} F$ and the fact that we could choose such an $\alpha$ to have support on $J^{k} F$ that intersects compactly the image of the prolongation $j^{k} G(M) \subset J^{k} F$ of a certain metric $G$. Natural questions arise. Are there more local and gauge invariant observables that could be defined in the same way? Are there sufficiently many such observables to separate points ${ }^{+}$on the physical phase space $\overline{\mathscr{P}}$ of GR?

The general mathematical context in which the answers must be sought is known as differential invariant theory [26, 30, 25]. Classical invariant theory is concerned with identifying functions on a $\mathscr{G}$-space (a space with an action of a group $\mathscr{G}$ ) that are invariant under the $\mathscr{G}$-action, these are the usual invariants. On the other hand, differential invariant theory, is concerned with fiber preserving group actions (more generally pseudogroup or groupoid actions) on the total space of a bundle, like our field vector bundle $F \rightarrow M$, and the actions induced on $J^{k} F \rightarrow M$ by prolongation. Then, differential invariants (of order $k$ ) are functions on $J^{k} F \rightarrow M$ that are invariant under the group action. For our purposes, the field bundle of metrics is $F=S^{2} T^{*} M$ and the group is $\mathscr{G}=\operatorname{Diff}(M)$, consisting of diffeomorphisms $\chi: M \rightarrow M$, and acting by pullback $\chi^{*}: F \rightarrow F$. Differential invariants are then precisely the socalled curvature scalars, that is, scalar functions tensorially constructed out of the metric, the Riemann curvature tensors and its covariant derivatives. For example, the $b^{i}$ defined in Equation (19) are differential invariants of order $k=2$. There are two ways of looking at differential invariants: algebraically and geometrically. Most structural results are proven from the algebraic perspective. On the other hand, it is easier to see from the geometric perspective how to construct local gauge invariant observables similar to the example of Equation (20).

The main structural algebraic result that we would like to mention is the socalled Lie-Tresse theorem, which dates back to the end of the 19th century, but was established in its global form only rather recently (see [25] and the references therein). This theorem is an analog of the finite generation results, originally due to Hilbert, in classical invariant theory [29]. For differential invariants, in addition to algebraic operations, we also need to allow differentiation to generate differential invariants of arbitrary orders from finite data. Before stating the result, let us recall the geometric formulation of differential equations in terms of jets, cf. [23, Apx.B] for more details and references. A differential equation of order $m \geq 0$, is usually specified in equational form, $P[\psi]=0$, where $P: \Gamma(F) \rightarrow \Gamma(E)$ a possibly non-linear

\footnotetext{
+ A set of functions separates the points of a space if, for each pair of points, there exist at least one function that takes on different values at these points.
} 
differential operator of order $m$ that takes sections of a bundle $F \rightarrow M$ as arguments and output sections of some other vector bundle $E \rightarrow M$. Essentially equivalently, we can specify a differential equation of order $m$ as submanifold $\mathcal{E} \subseteq J^{m} F$. Roughly, the set of all $m$-jets that satisfy $P=0$ constitutes the subset $\mathcal{E}$ and inversely, any bundle map $P: J^{m} F \rightarrow E$ that is zero only on $\mathcal{E} \subseteq J^{m} E$ defines the corresponding differential operator. A particular example could be $\mathcal{E}=J^{m} F$, which corresponds to the trivial equation $0=0$. A differential equation $\mathcal{E} \subseteq J^{m} F$ has natural prolongations $\mathcal{E}^{(k)} \subseteq J^{k} F$ for all $k \geq m$, which corresponds to taking into account all equations of the form $\partial_{i_{1}} \cdots \partial_{i_{k-m}} P[\psi]=0$ implied by $P[\psi]=0$. The following result is a rough restatement (sufficient for the purposes of this note) of the precise results of Theorems 1 and 2 of [25].

Proposition 4.1 (Lie-Tresse). Consider a differential equation $\mathcal{E} \subseteq J^{k} F$ with gauge symmetry, ${ }^{*}$ defined on a field bundle $F \rightarrow M$, with the action of gauge symmetries naturally prolongued to $J^{k} F \rightarrow M$. Assume that the equation and the gauge symmetry action satisfies a specific global algebro-geometric regularity condition (which is in fact satisfied by GR with diffeomorphisms as gauge symmetries). Then, there exists a finite order $l \geq 0$, a finite number of differential invariants (those left invariant by gauge transformations) $I_{j}$ on $J^{l} F$, and a finite number of invariant differential operators $D_{i}$ (such an operator acting on an invariant yields another invariant) such that any polynomial differential invariant of an arbitrary order $k \geq 0$ can be expressed as a polynomial in the generators $I_{j}$, possibly repeatedly differentiated by the $D_{i}$. Finally, for arbitrary order $k \geq 0$, the differential invariants separate the orbits of the gauge symmetry on a dense open subset $\mathcal{E}^{(k)} \subseteq \mathcal{E}^{(k)}$ consisting of generic orbits.

More geometrically, we can look at differential invariants as follows. Consider the quotient spaces $\sharp \mathcal{M}^{k}=J^{k} F / \operatorname{Diff}(M)$, known as the moduli spaces of $k$-jets of metrics on $M$ [17], with the projections denoted by $\mu_{k}: J^{k} F \rightarrow \mathcal{M}^{k}$. Clearly, differential invariants are precisely the smooth functions on $J^{k} F$ that come from the pullback of continuous functions on $\mathcal{M}^{k}$, those that belong to $C^{\infty}\left(J^{k} F\right) \cap \mu_{k}^{*}\left[C\left(\mathcal{M}^{k}\right)\right]$. If $\mathcal{M}^{k}$ were a manifold, it would be sufficient to consider $C^{\infty}\left(\mathcal{M}^{k}\right)$ instead of $C\left(\mathcal{M}^{k}\right)$. However, while $\mathcal{M}^{k}$ is well-defined as a topological space, it is only a manifold on a dense open subset [17], say $\mathcal{M}^{k} \subset \mathcal{M}^{k}$. Outside $\mathcal{M}^{k}, \mathcal{M}^{k}$ contains orbifold-type singularities, which correspond to jets of metrics admitting non-trivial isometries. A further complication is that $\mathcal{M}^{k}$ is in general not Hausdorff. This means that there exist jets of metrics that cannot be distinguished by continuous scalar curvature invariants alone. This phenomenon is particular to Lorentzian (and other pseudo-Riemannian) metrics and is absent when consideration is restricted to only Riemannian metrics. The failure of the Hausdorff property can be traced back to the non-compactness of the orthogonal group in Lorentzian signature [17].

To connect the algebraic and geometric points of view, consider Einstein's equations prolonged to an arbitrary order $k \geq 2$ and represented as a submanifold $\mathcal{E}^{(k)} \subset J^{k} F$. Clearly, $\mathcal{E}^{(k)}$ is invariant under diffeomorphisms and so projects to $\mu_{k}: \mathcal{E}^{(k)} \rightarrow \mathcal{R}^{k} \subseteq \mathcal{M}^{k}$, with $\stackrel{\circ}{\mathcal{R}}^{k}=\mathcal{R}^{k} \cap \dot{\mathcal{M}}^{k}$ a submanifold of $\dot{\mathcal{M}}^{k}$. The polynomial differential invariants mentioned in Proposition 4.1 are then functions on $\mathcal{R}^{k}$ and in fact separate the points of $\stackrel{\circ}{\mathcal{R}}^{k}$ and, by the Stone-Weierstrass theorem, generate $C^{\infty}\left(\stackrel{\circ}{\mathcal{R}}^{k}\right)$ by limits uniformly converging on compact sets.

* In the language of [25], this means that the equation is invariant under a pseudogroup action. $\sharp$ At the moment, we are not making a notational distinction, but we are really only interested in the subset of $J^{k} F$ corresponding to the jets of Lorentzian metrics, thus excluding degenerate metrics and metrics of other signatures. 
Now we come to the main observation that prompted this note. The connection between differential invariants and local observables in the generalized sense of Section 3 is most clearly seen with the help of the manifold $\mathcal{M}^{k}$. Namely, consider an $n$-form $\beta \in \Omega^{n}\left(\dot{\mathcal{M}}^{k}\right)$ with compact support and the horizontal density $\alpha \in \Omega^{n, 0}(F)$ obtained by the horizontal projection of the pullback of $\beta, \alpha=\mathrm{h}\left[\mu_{k}^{*} \beta\right]$. Letting $\mathscr{U} \subset \mathscr{C}$ be the subset of all metrics $G: M \rightarrow F$ such that $j^{k} G(M) \cap \operatorname{supp} \alpha$ is compact, we can define a local and gauge invariant observable with domain of definition $\mathscr{U}$ by the usual formula

$$
A[G]=\int_{M}\left(j^{k} G\right)^{*} \alpha .
$$

It is clearly gauge invariant, since by construction $\left(p^{k} \chi^{*}\right)^{*} \alpha=\alpha$. Further, it is clearly local in the generalized sense of Section 3, provided that $\mathscr{U}$ is open and non-empty. These properties do hold because of the following

Theorem 4.2. Given a non-empty compact set $K \subset \dot{\mathcal{M}}^{k}$, there exists a metric $G \in \mathscr{C}$ such that $\left(\mu_{k} \circ j^{k} G\right)^{-1}(K) \subseteq M$ is non-empty and compact. Further, such a metric $G$ has an open neighborhood $\mathscr{U} \subset \mathscr{C}$ (in the strong topology) such that $\mu_{k} \circ j^{k} H(M) \cap K$ is compact for each $H \in \mathscr{U}$.

Proof. First, we deal with the statement about existence. Let us ignore for the moment issues that might arise from non-trivial topology of $M$ and assume that $M \cong \mathbb{R}^{n}$, with some fixed global coordinate system. Let $\eta: M \rightarrow F$ be the standard Minkowski metric in those global coordinates. Take a point $r \in K \subset \mathcal{M}^{k}$, a point $x \in M$ and an open neighborhood $U \subset M$ of $x$ with compact closure. By construction, there is a jet $p \in J_{x}^{k} F$ such that $\mu_{k}(p)=r$. Consider the closed set $Q=(M \backslash U) \cup\{x\}$. Define $G_{Q}^{k}: Q \rightarrow J^{k} F$ so that $G_{Q}^{k}(x)=p \in J_{x}^{k} F$ and $G_{Q}^{k}(y)=j_{y}^{k} \eta \in J_{y}^{k} F$ for any $y \neq x$. By the Whitney extension theorem $[24, \S 22]$, there exists a metric $G \in \mathscr{C}$ such that $\left.j^{k} G(x)\right|_{Q}=G_{Q}^{k}$, which we can choose to be everywhere Lorentzian (non-degenerate). Thus, $\mu_{k} \circ j^{k} G$ and $K$ have at least the point $r$ in common. On the other hand, by construction, the pre-images $\left(\mu_{k} \circ j^{k} G\right)^{-1}(K) \subset\left(\mu_{k} \circ j^{k} G\right)^{-1}\left(\mathcal{M}^{k}\right) \subset M$ must be contained in $\bar{U}$, which is compact. Hence, the pre-image of $K$ must be compact, since it is closed and contained in $\bar{U}$. The same argument can be adapted without much difficulty to the case when $M$ has more complicated topology.

Second, we deal with the statement about an open neighborhood of $G \in \mathscr{C}$, which was constructed above. The following argument echos the proof of Theorem 3.1. We will define $\mathscr{U}=\left\{H \in \mathscr{C} \mid j^{k} H(M) \subset U\right\}$, for some to be determined open neighborhood $U \subset F$ of $j^{k} G(M)$. Obviously $G \in \mathscr{U}$ and $\mathscr{U}$ would be open in the strong topology. We build $U$ as the pre-image of an open set $V \subseteq M \times \mathcal{M}^{k}$ with respect to the map $\left(\pi^{k}, \mu_{k}\right): J^{k} F \rightarrow M \times \mathcal{M}^{k}$. If $V$ is an open neighborhood of the graph of $\mu_{k} \circ j^{k} G: M \rightarrow \mathcal{M}^{k}$, then $U$ is an open neighborhood of $j^{k} G(M)$. The way we constructed $G$ above, the intersection $I$ of the set $M \times K$ with the graph of $\mu_{k} \circ j^{k} G$ is compact. Take an open neighborhood $V^{\prime}$ with compact closure of $I$ and let $V=M \times\left(\mathcal{M}^{k} \backslash K\right) \cup V^{\prime}$. Thus, if $H \in \mathscr{U}$, the intersection of the graph of $\mu_{k} \circ j^{k} H$ with $M \times K$ must be confined to $V^{\prime}$, which has compact closure, and hence be compact. The last statement is equivalent to the pre-image $\left(\mu_{k} \circ j^{k} H\right)^{-1}(K) \subset M$ being compact, which concludes the proof.

Note that a direct application of the above theorem to the compact supp $f$ appearing in the definition of the functional $A[G]$ given by Equation (20), interpreted 
as a subset of $\dot{\mathcal{M}}^{2}$, establishes the claimed existence of a non-empty open domain $\mathscr{U} \subseteq \mathscr{C}$, making $\left.A\right|_{\mathscr{U}}$ a generalized local observable.

While we have concentrated on the case of gravitational theories, whose group of gauge transformations consists of diffeomorphisms, this method of defining gauge invariant local observables happens to reproduce the set of local observables for theories without gauge symmetries (the group of gauge symmetries is trivial) and those with gauge theories with gauge transformations that do not move points. Examples of the latter include the Maxwell and Yang-Mills theories. In the Maxwell theory, the basic differential invariant is the field strength. In the Yang-Mills case, the basic differential invariants are the compositions of the Lie algbra valued curvature forms composed with invariant polynomials on the Lie algebra. Smearing these basic invariants (or derivatives thereof) with compactly supported test functions reproduces the well-known standard local and gauge invariant observables in these theories [1].

We conclude this section by coming back to this natural question: are there enough local and gauge invariant observables in GR to separate the points of $\mathscr{C}$ ? In a sense, the answer is No, because we have already discussed above the fact that certain metrics cannot be distinguished by local curvature scalars. Further, some metrics may be resistant to belonging to the domain of definition $\mathscr{U}$ of any generalized local observable $\left.A\right|_{\mathscr{U}}$. This may happen when $M$ is non-compact and a metric $G$ possesses a region $U \subseteq M$ such that nearly isometric copies of $\left.G\right|_{U}$ repeat infinitely often throughout $M$ (a kind of almost periodic property). There is essentially no obstacle to engineering a gauge invariant local density $\alpha$ on $J^{k} F$ such that $\left(j^{k} G\right)^{*} \alpha$ has compact support in $U$, but it will likely also have support within any region nearly isometric to $\left.G\right|_{U}$, thus making the integral over $M$ ill defined. However, these are the only obstacles. We need to introduce a natural but somewhat technical condition on metrics that avoid these difficulties.

First, we say that a map $\nu: M \rightarrow N$ is image proper $\dagger \dagger$ if there exists an open set $N_{0} \subseteq N$ such that $\nu(M) \subseteq N_{0}$ and $\nu: M \rightarrow N_{0}$ is proper (the pre-image of any compact set is compact). Any proper map is image proper, since we can just choose $N_{0}=N$. On the other hand, any embedding is image proper, even if it is not proper, with any tubular neighborhood fulfilling the role of $N_{0}$. Let us say that two metrics $G_{1}, G_{2} \in \mathscr{C}$ can be distinguished by curvature scalars if there exists a $k \geq 0$ such that $\gamma_{i}=\mu_{k} \circ j^{k} G_{i}: M \rightarrow \mathcal{M}^{k}$ are image proper and the images $\gamma_{1}(M) \cap \dot{\mathcal{M}}^{k}$ and $\gamma_{2}(M) \cap \mathcal{M}^{k}$ do not coincide as subsets of $\mathcal{M}^{k}$.

Theorem 4.3. For any two metrics $G_{1}, G_{2} \in \mathscr{C}$ that can be distinguished by curvature scalars, there exists a local functional $A[G]$ defined on a domain $\mathscr{U} \subseteq \mathscr{C}$ (open in the strong topology) such that both $G_{1}, G_{2} \in \mathscr{U}$ and $A\left[G_{1}\right] \neq A\left[G_{2}\right]$.

Proof. By hypothesis, there is a $k \geq 0$ and a point $r \in \mathcal{M}^{k}$ such that, say, $r \in \gamma_{1}(M)=$ $\mu_{k} \circ j^{k} G_{1}(M)$ but $r \notin \gamma_{2}(M)=\mu_{k} \circ j^{k} G_{2}(M)$. Take a $\beta \in \Omega^{n}\left(\mathcal{M}^{k}\right)$ with compact support such that $r \in \operatorname{supp} \beta$ but $\gamma_{2}(M) \cap \operatorname{supp} \beta=\varnothing$. Let $A[G]=\int_{M}\left(\mu_{k} \circ j^{k} G\right)^{*} \beta$. Since the map $\gamma_{1}$ is image proper, we can always choose $\beta$ so that $\operatorname{supp} \beta$ is small enough to have compact intersection with $\gamma_{1}(M)$ and so that $A\left[G_{1}\right] \neq 0$. On the other hand, by construction, $A\left[G_{2}\right]=0$. Finally, since both $\gamma_{i}(M)$ have compact intersection with $\operatorname{supp} \beta$ (one of the intersections being empty), by Theorem 4.2, there exist (in the strong topology) open neighborhoods $\mathscr{U}_{1}$ and $\mathscr{U}_{2}$ of $G_{1}$ and $G_{2}$, respectively, such that $\mu_{k} \circ j^{k} G(M) \cap \operatorname{supp} \beta$ is also compact for each $G \in \mathscr{U}=\mathscr{U}_{1} \cup \mathscr{U}_{2}$. Clearly, $A[G]$ is well defined on $\mathscr{U}$ and $G_{1}, G_{2} \in \mathscr{U}$.

$\dagger \dagger$ Cf. [20, Exr.2.4.13], where this concept is used but not named. 


\section{Linearization and Poisson brackets}

Once a class of gauge invariant observables has been defined, as was done in Section 4, we would like to compute Poisson brackets between them. In general, neither the product nor the Poisson bracket of two local observables is a local observable (instead it is bilocal, with distributional smearing in case of the Poisson bracket) and the same is true for local observables in the generalized sense. It is an important and nontrivial question to decide on a minimal physically reasonable class of observables that is closed both under multiplication and Poisson brackets. The answer is essentially a class of multilocal observables with distributional smearings, which satisfy a certain microlocal spectral condition, which is discussed in more detail in [6, 8]. Below, we shall not be concerned with these details and instead content ourselves with a gauge invariant formula for the Poisson bracket of two local and gauge invariant observables.

As discussed extensively in [21, 23], what is usually known as the canonical Poisson bracket on the physical phase space $\overline{\mathscr{P}}$ can be equivalently expressed using the so-called Peierls formula (or Peierls bracket). The Peierls formula actually defines a Poisson bracket not only on $C^{\infty}(\overline{\mathscr{P}})$, but also extends it to $C^{\infty}(\mathscr{P})$ and even $C^{\infty}(\mathscr{C})$. This extension is not unique and is influenced, for instance, by the choice of gauge fixing. However, the restriction of the formula to $C^{\infty}(\overline{\mathscr{P}})$ is unique. The computation of the value of the Poisson bracket $\{A, B\}[G]$ of arbitrary observables $A$ and $B$ at a particular point (or gauge equivalence class of field configurations) $G \in \overline{\mathscr{P}}$ of a non-linear field theory reduces to the computation of the Poisson bracket of linear observables $\dot{A}_{G}$ and $\dot{B}_{G}$ in the linear theory obtained by linearization about $G$. Consider the linearized perturbation $H$ of the metric $G$. The relation between non-linear observables and linearized observables is

$$
A[G+\lambda H]=A[G]+\lambda \dot{A}_{G}[H]+O\left(\lambda^{2}\right) .
$$

In the case of a local observable $A[G]=\int_{M}\left(j^{k} G\right)^{*} \alpha$, the linearized observable is also local, $\dot{A}_{G}[H]=\int_{M} \dot{\alpha}[H]$, where $\dot{\alpha}$ is a density-valued differential operator defined by

$$
\left(j^{k}(G+\lambda H)\right)^{*} \alpha=\left(j^{k} G\right)^{*} \alpha+\lambda \dot{\alpha}_{G}[H]+O\left(\lambda^{2}\right) .
$$

We can define similarly $B[G]=\int_{M}\left(j^{k} G\right)^{*} \beta$ and $\dot{B}_{G}[H]=\int_{M} \dot{\beta}_{G}[H]$.

It is also useful to consider the formal adjoint differential operators $\dot{\alpha}_{G}^{*}$ and $\dot{\beta}_{G}^{*}$ defined by the existence of form-valued bidifferential operators $W_{\alpha}$ and $W_{\beta}$ such that

$$
f \dot{\alpha}_{G}[H]-\dot{\alpha}_{G}^{*}[f] \cdot H=\mathrm{d} W_{\alpha}[f, H] \quad \text { and } \quad f \dot{\beta}_{G}[H]-\dot{\beta}_{G}^{*}[f] \cdot H=\mathrm{d} W_{\beta}[f, H]
$$

for arbitrary $f \in C^{\infty}(M)$ and $H \in \Gamma(F)$, with the adjoint operators valued in the densitized dual bundle $\tilde{F}^{*}=F^{*} \otimes \Lambda^{n} M$. Let $\mathscr{U} \subseteq \overline{\mathscr{P}}$ be a common domain on which $A$ and $B$ are defined and let $G \in \mathscr{U}$. Then, by the generalized locality property, $\dot{\alpha}_{G}[H]$ and $\dot{\beta}_{G}[H]$ have compact support for arbitrary $H$. It is then not hard to see that all of $\dot{\alpha}_{G}^{*}[1], W_{\alpha}[1, H], \dot{\beta}_{G}^{*}[1]$ and $W_{\beta}[1, H]$ will also have compact support for arbitrary $H$. Therefore, an application of Stokes' lemma gives us the identities

$$
\dot{A}_{G}[H]=\int_{M} \dot{\alpha}_{G}^{*}[1] \cdot H \quad \text { and } \quad \dot{B}_{G}[H]=\int_{M} \dot{\beta}_{G}^{*}[1] \cdot H
$$

The Peierls formula for the Poisson bracket of observables of the form in Equation (25) was considered explicitly in [23, Sec.4.4] (see also [13] and [18, Ex.3.8]) and is given 
by the formula

$$
\{A, B\}[G]=\left\{\dot{A}_{G}, \dot{B}_{G}\right\}_{G}=\int_{M \times M} \dot{\alpha}_{G}^{*}[1](x) \cdot E_{G}(x, y) \cdot \dot{\beta}_{G}^{*}[1](y)
$$

where $E_{G}(x, y)=E_{G}^{+}(x, y)-E_{G}^{-}(x, y)$, with $E_{G}^{ \pm}(x, y)$ being the integral kernels of the retarded and advanced Green functions of the so-called Lichnerowicz operator (which is a hyperbolic differential operator obtained from a de Donder gauge fixing of the linearized Einstein equations) of the background metric $G$.

The result is gauge invariant, that is $\{A, B\}\left[\chi^{*} G\right]=\{A, B\}[G]$ for a diffeomorphism $\chi^{*}: M \rightarrow M$, essentially by construction. More explicitly, since each of the elements in the formula is invariantly constructed from the metric $G$, the following identities hold: $\dot{\alpha}_{\chi^{*} G}^{*}=\chi^{*} \dot{\alpha}_{G}^{*}, \dot{\beta}_{\chi^{*} G}^{*}=\chi^{*} \dot{\beta}_{G}^{*}$ and $E_{\chi^{*} G}(x, y)=(\chi, \chi)^{*} E_{G}(x, y)$, where $(\chi, \chi)^{*}: M \times M \rightarrow M \times M$ is defined in the obvious way. Combining these identities with formula (26) explicitly shows that $\{A, B\}$ is a gauge invariant (though now distributional bilocal, instead of local) observable.

It is also worth examining whether the linearized observable $\dot{A}_{G}[H]$ fits the criteria of being a gauge invariant observable for linearized gravity on the background $G$. The answer is of course Yes, as follows from the identity $\mathcal{L}_{v} \alpha[G]=\dot{\alpha}\left[\mathcal{L}_{v} G\right]$, where $\mathcal{L}_{v}$ is the Lie derivative with respect to a vector field $v$, which is the linearized version of the invariance property $\chi^{*} \alpha[G]=\alpha\left[\chi^{*} G\right]$, and the Cartan magic formula $\mathcal{L}_{v} \alpha[G]=\mathrm{d}\left(\iota_{v} \alpha[G]\right)$ for top-degree forms. For convenience, let us also define the differential operator $K_{G}[v]=\mathcal{L}_{v} G$, which we will call the Killing operator. The gauge invariance condition for $\dot{A}_{G}[H]$ in linearized gravity consists in the requirement that $\dot{A}_{G}\left[K_{G}[v]\right]=0$ for any vector field $v$. This follows from the preceding identities:

$$
\dot{A}_{G}\left[K_{G}[v]\right]=\int_{M} \dot{\alpha}_{G}\left[\mathcal{L}_{v} G\right]=\int_{M} \mathcal{L}_{v} \alpha[G]=\int_{M} \mathrm{~d}\left(\iota_{v} \alpha[G]\right)=0,
$$

where the last equality follows from the fact that $\iota_{v} \alpha[G]$ has compact support by the locality hypothesis. Thus, $\dot{A}_{G}[H]$ is a linear, local and gauge invariant observable in linearized gravity.

Let us recall the notion of linear, local and gauge invariant observable from [13] (also [23, Sec.4.4], [18, Ex.3.8]), which is an observable of the form

$$
C[H]=\int_{M} \gamma \cdot H
$$

with a compactly supported section $\gamma: M \rightarrow \tilde{F}^{*}$ that satisfies the condition $K_{G}^{*}[\gamma]=0$, where $K_{G}^{*}$ is the formal adjoint of the Killing operator $K_{G}$. More explicitly, there exists a form-valued bidifferential operator $W_{K}$ such that $\gamma \cdot K_{G}[v]-K_{G}^{*}[\gamma] \cdot v=\mathrm{d} W_{K}[\gamma, v]$ for any vector field $v$ and any section $\gamma: M \rightarrow \tilde{F}^{*} ; K_{G}^{*}$ is equivalent to the divergence of a symmetric 2-tensor.

Proposition 5.1. Given the linearized observable $\dot{A}_{G}[H]$, as discussed above, there always exists a local observable $C[H]$ in linearized gravity of the form (28) such that $\dot{A}_{G}[H]=C[H]$.

Proof. For this result to hold, it is clearly sufficient that there exist a compactly supported section $\gamma: M \rightarrow \tilde{F}^{*}$, satisfying $K_{G}^{*}[\gamma]=0$, and a form-valued linear differential operator $\mu[H]$, with compact support for arbitrary argument $H: M \rightarrow F$, such that $\dot{\alpha}_{G}[H]=\gamma \cdot H+\mathrm{d} \mu[H]$. We shall construct such $\gamma$ and $\mu[H]$ explicitly. 
Recall the identity $\dot{\alpha}_{G}[H]=\dot{\alpha}_{G}^{*}[1] \cdot H+\mathrm{d} W_{\alpha}[1, H]$. We set $\mu[H]=W_{\alpha}[1, H]$ and $\gamma=\dot{\alpha}_{G}^{*}[1]$. It remains to show that $K_{G}^{*}\left[\dot{\alpha}_{G}^{*}[1]\right]=0$. Note that, from the gauge invariance of $\dot{A}_{G}[H]$ discussed earlier, we already know that

$$
\begin{aligned}
\dot{\alpha}_{G}^{*}[1] \cdot K_{G}[v] & =\dot{\alpha}_{G}\left[K_{G}[v]\right]+\mathrm{d} W_{\alpha}\left[1, K_{G}[v]\right] \\
& =\mathrm{d}\left(\iota_{v} \alpha[G]+W_{\alpha}\left[1, K_{G}[v]\right]\right)
\end{aligned}
$$

for an arbitrary vector field $v$. On the other hand, we also have the equality

$$
\dot{\alpha}_{G}^{*}[1] \cdot K_{G}[v]=K_{G}^{*}\left[\dot{\alpha}_{G}^{*}[1]\right] \cdot v+\mathrm{d} W_{K}\left[\dot{\alpha}_{G}^{*}[1], v\right] .
$$

The final tool that we need to invoke is the well-known fact [28, Thm.4.7] that, for any top-degree form valued linear differential operator $\psi[v]$, in any decomposition of the form $\psi[v]=\phi \cdot v+\mathrm{d} \xi[v]$ the coefficients $\phi$ and the term $\mathrm{d} \xi[v]$ are unique (in particular $\phi=\delta_{E L}[\psi[v]]$ is the Euler-Lagrange derivative of $\left.\psi[v]\right)$. Thus, comparing Equations (29) and (30), we find that $K_{G}^{*}\left[\dot{\alpha}_{G}^{*}[1]\right]=0$, as was desired.

\section{Discussion}

In this note, we have discussed the notion of local observables in field theory, advocating that the standard notion of locality (Section 2) should be relaxed in a well-defined way (Section 3). We have argued that the two motivating properties of local observables, diffusion of UV singularities and IR regularization, still hold for generalized local observables in the sense defined in Section 3.

A small price to pay is that a generalized local observable may be naturally defined as functions only on an open subset $\dagger$ of the full phase space of the field theory. Classically, it is no problem to restrict one's attention to an open subset of the full phase space. If needed, such an observable may be extended to the full phase space by appealing to basic results in differential topology. We have shown that linearization about a specific point of the configuration space gives a gauge invariant observable for linearized gravity on the corresponding background, irrespective of how large is the neighborhood of the linearization point on which the observable can be defined. That is of course the expected result for the linearization of an observable invariant under full non-linear gauge transformations. We expect the same behavior at any order of perturbation theory; the truncated expansion of the observable should be invariant under perturbative gauge transformations truncated at the same order, which is sufficient for the purposes of perturbative quantization.

It is well-known that gravitational theories do not admit any non-trivial local observables that are also gauge-invariant. Hence, it is a significant advantage of the new definition that the class of generalized local observables in gravitational theories does admit a large number of observables that are gauge invariant (Section 4). We have given a typical example of one such observable, motivated by an old proposal of Komar and Bergmann [2,3]. In fact, such gauge invariant observables are sufficient to separate the gauge orbits on a large open subset of the phase space (Theorems 4.2 and 4.3).

$\dagger$ A related mathematical phenomenon occurs in complex and algebraic geometry. Certain complex and algebraic varieties have very few globally defined functions. By restricting to open subsets, many more functions can be considered, that otherwise developed singularities if extended to the entire space. Such partially defined functions are studied in the theory of sheaves. We have not developed this analogy in detail because there is not yet a clear application of sheaf theory in this context, other than as a concise terminology. 
The main technical tool in the construction of these gauge invariant observables is the theory of differential invariants, which in the literature on GR are also known as curvature scalars or curvature invariants.

Unfortunately, the large open subset of the phase space mentioned above specifically excludes solutions that have a high degree of symmetry. Some of these symmetric solutions can be of great physical importance, at least in GR, with examples like Minkowski or Schwarzschild or de Sitter spacetimes. The reason for the exclusion is that observables based on curvature scalars are incapable of separating certain inequivalent gauge equivalence classes of solutions. At the geometric level, the same phenomenon manifests itself in the fact that the moduli space of Lorentzian metrics (the quotient of jets of Lorentzian metrics by the action of diffeomorphisms) is not Hausdorff [17]. A well-known example is that all curvature scalars vanish both on flat Minkowski spacetime as well on non-flat null pp-wave spacetimes (non-linear wave gravitational wave solutions) $[19,10,11]$. This is problematic if one would like to connect perturbative theory about Minkowski space with non-linear local observables of the kind discussed above. In principle, it is known that there exist nonscalar differential invariants that are capable of locally distinguishing non-isometric Lorentzian metrics (cf. the Cartan-Karlhede algorithm discussed in [33, Ch.9] and references therein). At this point it remains an open problem to be investigated whether these more refined differential invariants could be used to construct local (or perhaps multilocal) observables that are capable of separating all gauge orbits on the phase space of GR and other gravitational theories.

In Section 5, we showed that generalized local and gauge invariant observables have gauge invariant Poisson brackets using the Peierls formula. However, Poisson brackets of local observables are in general no longer local. At best they could be described as multilocal with distributional smearings. Such observables have been previously discussed in the literature [6, 8], with careful attention paid to the class of distributions that can be consistently allowed to construct an algebra of multilocal observables closed under Poisson brackets. The added complication in gravitational theories, as is evident from the Peierls formula, is that in order to preserve gauge invariance we must allow distributional smearings themselves to depend on the metric and possibly other dynamical fields. Thus, another important avenue for investigation is the generalization of multilocal observables to allow for field-dependent distributional smearings.

It might be argued that the local and gauge invariant observables that we have introduced in this note are of a relational kind (see [34] and references therein). However, they do not automatically come with a phenomenological interpretation. That is, given a particular observable of this kind, it may not be immediately clear what kind of experimental protocol would be modeled by it (this issue is discussed clearly in [22]). On the other hand, there is some existing literature that has considered relational observables in linearized and perturbative gravity with more clear phenomenological interpretations, but ran into UV divergences in explicit computations $[14,39,5,35,38,36,27,22,4]$. Perhaps replacing the overly singular proposed observables in these references with regularized versions written as local and gauge invariant observables would yield a double benefit: provide certain local observables with phenomenological interpretations, diffuse UV singularities in explicit computations. As a further step, it would be most interesting to identify local gauge invariant observables that would model some aspects of the data collected by cosmological observations, such as the Cosmic Microwave Background temperature 
fluctuations and its polarization.

It should also be mentioned that another attempt [16] to write down relational observables (though without clear phenomenological interpretations) using curvature scalars ran into IR divergences in explicit computations. On the other hand, our local observables are designed to be IR regularizing and might give better results in similar computations.

\section{Acknowledgments}

The author would like to thank Valentin Lychagin for helpful discussions on the topic of differential invariants. Thanks also to Jochen Zahn for comments on an earlier version of the manuscript. The kind hospitality of the Department of Mathematics at the University of York, where part of the manuscript was completed, is also acknowledged.

\section{References}

[1] G. Barnich, F. Brandt, and M. Henneaux, "Local BRST cohomology in gauge theories," Physics Reports 338 (2000) 439-569, arXiv:hep-th/0002245.

[2] P. Bergmann and A. Komar, "Poisson brackets between locally defined observables in general relativity," Physical Review Letters 4 (1960) 432-433.

[3] P. G. Bergmann, "Observables in general relativity," Reviews of Modern Physics 33 (1961) 510-514.

[4] B. Bonga and I. Khavkine, "Quantum astrometric observables. II. Time delay in linearized quantum gravity," Physical Review D 89 (2014) 024039, arXiv:1307.0256.

[5] J. Borgman and L. H. Ford, "Effects of stress tensor fluctuations upon focusing," Physical Review D 70 (2004) 064032, arXiv:gr-qc/0307043.

[6] R. Brunetti and K. Fredenhagen, "Quantum field theory on curved backgrounds," in Quantum Field Theory on Curved Spacetimes: Concepts and Methods, C. Bär and K. Fredenhagen, eds., vol. 786 of Lecture Notes in Physics. Springer, Berlin, 2009.

[7] R. Brunetti, K. Fredenhagen, and K. Rejzner, "Quantum gravity from the point of view of locally covariant quantum field theory," 2013. http://arxiv.org/abs/1306.1058v4.

[8] R. Brunetti, K. Fredenhagen, and P. L. Ribeiro, "Algebraic structure of classical field theory I: Kinematics and linearized dynamics for real scalar fields," 2012. http://arxiv.org/abs/1209.2148.

[9] D. Christodoulou and S. Klainerman, The global nonlinear stability of the Minkowski space, vol. 41 of Princeton Mathematical Series. Princeton University Press, Princeton, NJ, 1993.

[10] A. Coley, S. Hervik, and N. Pelavas, "Spacetimes characterized by their scalar curvature invariants," Classical and Quantum Gravity 26 (2009) 025013, arXiv:0901.0791.

[11] A. Coley, S. Hervik, and N. Pelavas, "Lorentzian manifolds and scalar curvature invariants," Classical and Quantum Gravity 27 (2010) 102001, arXiv:1003.2373.

[12] B. S. DeWitt, "The quantization of geometry," in Gravitation: An Introduction to Current Research, L. Witten, ed., ch. 8, pp. 266-381. John Wiley \& Sons, New York, NY, 1963.

[13] C. J. Fewster and D. S. Hunt, "Quantization of linearized gravity in cosmological vacuum spacetimes," Reviews in Mathematical Physics 25 (2013) 1330003, arXiv:1203.0261.

[14] L. H. Ford, "Gravitons and light cone fluctuations," Physical Review D 51 (1995) 1692.

[15] K. Fredenhagen and K. Rejzner, "Batalin-Vilkovisky formalism in the functional approach to classical field theory," Communications in Mathematical Physics 314 (2012) 93-127, arXiv: $1101.5112 \mathrm{v} 1$.

[16] S. Giddings, D. Marolf, and J. Hartle, "Observables in effective gravity," Physical Review D 74 (2006) 064018, arXiv:hep-th/0512200.

[17] A. Gordillo, J. Navarro, and J. B. Sancho, "Moduli spaces for finite-order jets of Riemannian metrics," Differential Geometry and its Applications 28 (2009) 672-688, arXiv:0808.2996.

[18] T.-P. Hack and A. Schenkel, "Linear bosonic and fermionic quantum gauge theories on curved spacetimes," General Relativity and Gravitation 45 (2013) 877-910, arXiv:1205.3484.

[19] S. Hervik and A. Coley, "Curvature operators and scalar curvature invariants," Classical and Quantum Gravity 27 (2010) 095014, arXiv:1002.0505.

[20] M. W. Hirsch, Differential Topology, vol. 33 of Graduate Texts in Mathematics. Springer, 1976. 
[21] I. Khavkine, "Characteristics, conal geometry and causality in locally covariant field theory," 2012. http://arxiv.org/abs/1211.1914.

[22] I. Khavkine, "Quantum astrometric observables: Time delay in classical and quantum gravity," Physical Review D 85 (2012) 124014, arXiv:1111.7127.

[23] I. Khavkine, "Covariant phase space, constraints, gauge and the Peierls formula," International Journal of Modern Physics A 29 (2014) 1430009, arXiv:1402.1282.

[24] A. Kriegl and P. W. Michor, The Convenient Setting of Global Analysis, vol. 53 of Mathematical Surveys and Monographs. American Mathematical Society, 1997.

[25] B. Kruglikov and V. Lychagin, "Global Lie-Tresse theorem," 2015. http://arxiv.org/abs/1111.5480v3.

[26] D. Krupka and J. Janyška, Lectures on differential invariants. Universita J.E. Purkyně, Brno, 1990.

[27] S. Ohlmeyer, The measurement of length in linear quantum gravity. PhD thesis, DESY, Hamburg, 1997. http://unith.desy.de/research/aqft/doctoral_theses/.

[28] P. J. Olver, Applications of Lie groups to differential equations, vol. 107 of Graduate Texts in Mathematics. Springer-Verlag, New York, second ed., 1993.

[29] P. J. Olver, Classical Invariant Theory, vol. 44 of London Mathematical Society Student Texts. Cambridge University Press, Cambridge, 1999.

[30] P. J. Olver and J. Pohjanpelto, "Differential invariant algebras of Lie pseudo-groups," Advances in Mathematics 222 (2009) 1746-1792.

[31] K. Rejzner, Batalin-Vilkovisky formalism in locally covariant field theory. PhD thesis, Hamburg, 2011. arXiv: 1111.5130.

[32] A. A. Sharapov, "Peierls brackets in non-Lagrangian field theory," International Journal of Modern Physics A 29 (2014) 1450157, arXiv:1408.2329.

[33] H. Stephani, D. Kramer, M. MacCallum, C. Hoenselaers, and E. Herlt, Exact Solutions of Einstein's Field Equations. Cambridge University Press, Cambridge, 2003.

[34] J. Tambornino, "Relational observables in gravity: a review," Symmetry, Integrability and Geometry: Methods and Applications 8 (2012) 017, arXiv: 1109.0740

[35] R. T. Thompson and L. H. Ford, "Spectral line broadening and angular blurring due to spacetime geometry fluctuations," Physical Review D 74 (2006) 024012, arXiv: gr-qc/0601137.

[36] N. C. Tsamis and R. P. Woodard, "Physical Green's functions in quantum gravity," Annals of Physics (N.Y.) $\mathbf{2 1 5}$ (1992) 96-155.

[37] C. Wockel, "A generalisation of Steenrod's approximation theorem," Archivum mathematicum (Brno) (2009) 9-104, arXiv:math/0610252. http://www.emis.de/journals/AM/09-2/am1753.html.

[38] R. P. Woodard, Invariant formulation of and radiative corrections in quantum gravity. $\mathrm{PhD}$ thesis, Harvard University, Cambridge, MA., 1984.

[39] H. Yu and L. H. Ford, "Light-cone fluctuations in flat spacetimes with nontrivial topology," Physical Review D 60 (1999) 084023, arXiv:gr-qc/9904082. 